\title{
THE ISOLATION AND IDENTIFICATION OF BACTEROIDES SPP. FROM THE NORMAL HUMAN VAGINAL FLORA
}

\author{
B. I. DUERDEN \\ Department of Medical Microbiology, University of Sheffield Medical School, \\ Beech Hill Road, Sheffield S10 $2 R X$
}

THE GR AM-NEG ATIVE anaerobic bacilli that form part of the normal commensal flora of the vagina and cervix have been the subject of several investigations, but the results have been conflicting. The presence of Bacteroides in the vagina was first reported by Burdon (1928) who isolated B. melaninogenicus from 28 out of 35 normal women. Mead and Louria (1969) and Suzuki and Ueno (1971, cited by Finegold 1977) found that Bacteroides were commonly present in the normal vaginal flora. Gorbach et al. (1973) isolated Bacteroides spp. from the cervical flora of $57 \%$ of 30 healthy women and Sanders et al. (1975) isolated Bacteroides spp. from endocervical cultures from $65 \%$ of 26 healthy women. However, Neary et al. (1973) recovered Bacteroides spp. from only $8.6 \%$ of vaginal swabs from 246 pre-operative gynaecological patients and Leigh, Kershaw and Simmons (quoted by Leigh, 1976) isolated Bacteroides from only $4.6 \%$ of 500 women attending a family-planning clinic and $5 \%$ of 200 patients attending a gynaecological outpatient clinic. Hurley et al. (1974) collected specimens of vaginal secretions from the posterior fornix of 280 unselected pregnant women and found Bacteroides spp. in only $15(5.4 \%)$ and Werner et al. (1978) isolated Bacteroides spp. from vaginal swabs from four out of 100 healthy pregnant women. In a quantitative study, Lindner, Plantema and Hoogkamp-Korstanje (1978) found that the mean $\log _{10}$ number of viable Bacteroides spp. per $\mathrm{ml}$ of vaginal secretions was 7-8 but they isolated Bacteroides spp. from only $4 \%$ of normal women, $1 \%$ of pregnant women and $28 \%$ of women with cervicitis.

The differences may reflect different methods of investigation. The vagina is not a single environment; the flora of the lower vagina is related to that of the perineum and introitus and differs from that of the cervix and fornices. Moreover, the physiological state of the vaginal mucosa and secretions changes with age, with the menstrual cycle and with pregnancy (Hurley et al., 1974).

The Bacteroides spp. isolated in most studies have not been identified and the relationship of these isolates to those from other sites is not clear. The present study was undertaken to determine the carriage of Bacteroides and identify the strains isolated from 20 normal healthy women attending a family-planning clinic. 


\section{MATERIALS AND METHODS}

One hundred and twenty strains isolated from 20 vaginal specimens were studied.

Anaerobic incubation. The anaerobic procedure of Collee et al. (1972) was followed in all essential aspects; incubation was at $37^{\circ} \mathrm{C}$ in an atmosphere of $\mathrm{H}_{2} 90 \%$ and $\mathrm{CO}_{2} 10 \%$. A slope of Simmons citrate medium seeded with Pseudomonas aeruginosa was included in each jar as a control.

Specimens. High vaginal swabs were collected from 20 normal healthy women and the swabs were immediately broken off into a vial of VMG II transport medium (Möller, 1966). The swabs were collected from the cervix and posterior fornix under direct vision during examination with a Sim's speculum and before manual examination. The subjects were women aged 20-40 years attending a family-planning clinic for routine vaginal examination and cervical cytology. Samples were not accepted from women who had any vaginal pathology or who were receiving antibiotic therapy. The specimens were processed on the day that they were collected.

Isolation of Bacteroides. Each swab was used to seed a sector of a plate of pre-reduced BM medium made selective for Bacteroides by the addition of kanamycin $75 \mu \mathrm{g} / \mathrm{ml}$ and vancomycin $2.5 \mu \mathrm{g} / \mathrm{ml}$ (see Duerden, 1980a). The plates were examined after incubation for $48 \mathrm{~h}$. All colony types were noted and five representative colonies were subcultured on to fresh BM agar without antibiotics. After re-incubation for a further $48 \mathrm{~h}$, additional colony types were noted and another five representative colonies were subcultured. The colony types were selected in approximate proportion to their comparative numbers on the primary isolation plate. When possible, 10 colonies were collected from each specimen; but this was not always possible because there was no growth of Bacteroides from seven of the specimens and fewer than 10 colonies were isolated from two specimens. The isolates were suspended in a freezing medium of Nutrient Broth No. 2 (Oxoid) with inactivated Horse Serum (Wellcome) $10 \%$ and glucose $1 \%$ and held at $-70^{\circ} \mathrm{C}$ in a liquid-nitrogen container.

Identification of isolates. The strains were identified by the methods of Duerden et al. (1976, 1980). The tests were: colony morphology after 48-h incubation on blood agar; cell morphology in gram-stained smears from 48-h cultures on blood agar and in BM broth with cooked-meat particles (see Deacon, Duerden and Holbrook, 1978); pigment production on BM agar; haemolysis on human blood agar; motility in BM broth; antibiotic-disk resistance tests with disks separately containing neomycin $1000 \mu \mathrm{g}$, kanamycin $1000 \mu \mathrm{g}$, penicillin 2 units and rifampicin 15 $\mu \mathrm{g}$; tolerance tests with taurocholate, deoxycholate, Victoria blue $4 \mathrm{R}$ and ethyl violet; biochemical tests for the production of indole, digestion of gelatin and hydrolysis of aesculin; and fermentation tests with glucose, lactose, sucrose, rhamnose, trehalose, mannitol and xylose.

\section{RESULTS}

Gram-negative, non-sporing, non-motile obligately anaerobic bacilli of the Bacteroides group were isolated from $13(65 \%)$ subjects and 120 isolates were selected for identification; 10 colonies were selected from each of the 11 primary plates that yielded a moderate or heavy growth of Bacteroides; eight colonies were obtained from one specimen and only two colonies from another. Complete sets of results were obtained with 113 isolates; seven isolates failed to grow on subculture from the preserved cultures. One hundred and two isolates representing 11 recognised species and subspecies were identified and the numbers of isolates allocated to each species are shown in table I. Six isolates were allocated to the $B$. fragilis group, 88 to the $B$. melaninogenicus/oralis group, 16 were non-saccharolytic, including nine pigmented $B$. asaccharolyticus isolates, and three isolates could not be allocated to a recognised species. 
TABLE I

Identification of vaginal isolates from 13 subjects

\begin{tabular}{|c|c|}
\hline Species & $\begin{array}{l}\text { Number of isolates } \\
\text { (and group total) }\end{array}$ \\
\hline $\begin{array}{l}\text { B. fragilis group } \\
\text { B. distasonis } \\
\text { B. vulgatus } \\
\text { B. thetaiotaomicron } \\
\text { B. splanchnicus }\end{array}$ & $\begin{array}{l}(6) \\
1 \\
3 \\
1 \\
1\end{array}$ \\
\hline 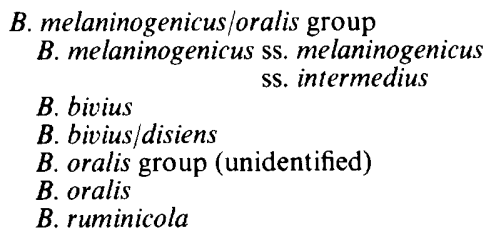 & $\begin{array}{r}(88) \\
14 \\
19 \\
16 \\
21 \\
1 \\
10 \\
7\end{array}$ \\
\hline $\begin{array}{l}\text { Asaccharolytic group } \\
\text { B. asaccharolyticus } \\
\text { Non-pigmented non-saccharolytic spp. }\end{array}$ & $\begin{array}{c}(16) \\
9 \\
7\end{array}$ \\
\hline Bacteroides spp. (unidentified) & (3) \\
\hline TOTAL & 113 \\
\hline
\end{tabular}

\section{B. fragilis group}

All the $B$. fragilis-group isolates except the $B$. distasonis strain were from one subject. The patterns of results obtained with the isolates were typical of the designated species except that the $B$. splanchnicus strain was tolerant of ethyl violet and two identical isolates of B. vulgatus were tolerant of deoxycholate.

\section{B. melaninogenicus/oralis group}

The most common species of Bacteroides isolated from the normal vaginal flora were members of the $B$. melaninogenicus/oralis group. The 88 isolates allocated to this group accounted for $78 \%$ of the Bacteroides isolated and identified in this study. All were inhibited by taurocholate, deoxycholate and ethyl violet, resistant to kanamycin and sensitive to rifampicin. Seventeen isolates were tolerant of Victoria blue 4R, 24 were resistant to penicillin and 12 isolates of B. melaninogenicus were resistant to the neomycin $1000 \mu \mathrm{g}$-disk.

The 19 isolates of $B$. melaninogenicus ss. intermedius readily produced pigmented colonies; they produced indole and fermented glucose but 11 isolates, including six identical isolates from one subject and three from another, were resistant to neomycin and 10 of them fermented glucose weakly after 4-7 days. Most of the 14 B. melaninogenicus ss. melaninogenicus isolates were typical of the subspecies but one isolate was resistant to the neomycin disk and six identical isolates from one subject were tolerant of Victoria blue 4R, resistant to penicillin and produced brown-pigmented colonies only slowly. 
The 37 isolates allocated to the $B$. bivius/disiens complex formed the largest group of vaginal isolates. The two species are distinguished by the results of tests for the fermentation of lactose but the significance of this test was not appreciated until the study was in progress and it was not included in the set of tests until the later stages of the investigation. All the isolates gave patterns of results typical of $B$. bivius and $B$. disiens; 10 isolates were tolerant of Victoria blue $4 \mathrm{R}$ and 18 were resistant to penicillin. Tests for the fermentation of lactose and sucrose were performed on 16 isolates in the later stages of the investigation; all fermented lactose but not sucrose and were, therefore, identified as B. bivius.

The $10 \mathrm{~B}$. oralis isolates gave patterns of results that were generally typical of the species; seven strains were resistant to penicillin and two identical isolates from one subject fermented mannitol. One isolate gave an intermediate pattern of results within the group and could not be identified. It fermented glucose, lactose and sucrose, like $B$. oralis, but did not hydrolyse aesculin. The seven isolates identified as $B$. ruminicola hydrolysed aesculin and fermented glucose and xylose. In addition two isolates fermented rhamnose and two identical isolates from one subject fermented rhamnose, trehalose and mannitol.

\section{Asaccharolytic group}

The 16 non-saccharolytic isolates included nine pigmented strains of $B$. asaccharolyticus; eight isolates gave patterns of results typical of the species but one did not produce indole. The seven non-pigmented non-saccharolytic strains gave three distinct patterns of results. Three isolates were similar to $B$. asaccharolyticus but did not produce pigment. A pair of isolates from one subject were inhibited in tolerance tests, resistant only to kanamycin and digested gelatin, but hydrolysed aesculin and did not produce indole. The remaining two isolates, from different subjects, were inhibited in the tolerance tests, sensitive to the four antibiotic disks and unreactive in other tests except for the digestion of gelatin.

Three fermentative isolates could not be identified; two identical isolates from one subject gave the tolerance and antibiotic-disk resistance patterns of the $B$. fragilis group, but they did not produce indole or hydrolyse aesculin and fermented only glucose. The third isolate gave the tolerance and antibiotic disk resistance patterns of the B. melaninogenicus/oralis group but it produced indole, hydrolysed aesculin and fermented glucose and mannitol.

\section{Identification of isolates from individual subjects}

When identical isolates from one subject were treated as a single strain, 56 strains were isolated from the 13 subjects and the identity of these strains is shown in table II. The distribution of species amongst the 13 subjects is shown in table III. The mean number of strains isolated from subjects for whom complete sets of results were obtained with more than six isolates was 4.6 
TABLE II

Strains of Bacteroides spp. isolated from 13 subjects

\begin{tabular}{|c|c|}
\hline Species & $\begin{array}{l}\text { Number of strains } \\
\text { isolated (and group total) }\end{array}$ \\
\hline $\begin{array}{l}\text { B. fragilis group } \\
\text { B. distasonis } \\
\text { B. vulgatus } \\
\text { B. thetaiotaomicron } \\
\text { B. splanchnicus }\end{array}$ & $\begin{array}{l}(5) \\
1 \\
2 \\
1 \\
1\end{array}$ \\
\hline 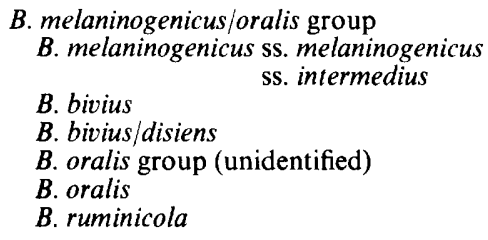 & $\begin{array}{c}(38) \\
5 \\
7 \\
4 \\
11 \\
1 \\
5 \\
5\end{array}$ \\
\hline $\begin{array}{l}\text { Asaccharolytic group } \\
\text { B. asaccharolyticus } \\
\text { Non-pigmented non-saccharolytic spp. }\end{array}$ & $\begin{array}{c}(11) \\
6 \\
5\end{array}$ \\
\hline Bacteroides spp. (unidentified) & $(2)$ \\
\hline TOTAL & 56 \\
\hline
\end{tabular}

(range 2-8). The subject that yielded eight strains was unusual because the eight strains included all except one of the $B$. fragilis-group isolates and only one isolate from the B. melaninogenicus/oralis group. At least one strain of the $B$. melaninogenicus/oralis group was isolated from each subject and one member of the group was often predominant but strains of the $B$. fragilis and asaccharolytic groups were detected in specimens from only a few subjects.

TABLE III

Species of Bacteroides isolated from vaginal swabs from 13 subjects

\begin{tabular}{|c|c|c|c|c|c|c|c|c|c|c|c|c|c|}
\hline \multirow[b]{2}{*}{ Species } & \multicolumn{13}{|c|}{ Isolation of the given species from subject number } \\
\hline & 1 & 2 & 3 & 4 & 5 & 6 & 7 & 8 & 9 & 10 & 11 & 12 & 13 \\
\hline $\begin{array}{l}\text { B. distasonis } \\
\text { B. vulgatus } \\
\text { B. thetaiotaomicron } \\
\text { B. splanchnicus }\end{array}$ & & & & & & & $\begin{array}{l}+ \\
+ \\
+\end{array}$ & + & & & & & \\
\hline B. melaninogenicus ss. melaninogenicus & & + & + & & & & & & + & & & + & \\
\hline $\begin{array}{ll}\text { B. bivius } & \text { ss. intermedius }\end{array}$ & & + & + & & + & + & & & & & & & + \\
\hline $\begin{array}{l}\text { B. bivius/disiens } \\
\text { B. oralis group (unidentified) } \\
B \text { oralis }\end{array}$ & + & & & $\begin{array}{l}+ \\
+\end{array}$ & & . & + & + & + & + & + & & + \\
\hline B. ruminicola & & & + & + & & & & & & & & + & + \\
\hline $\begin{array}{l}\text { B. asaccharolyticus } \\
\text { Non-pigmented non-saccharolytic spp. }\end{array}$ & & + & & + & & & $\begin{array}{l}+ \\
+\end{array}$ & + & & + & + & & + \\
\hline Bacteroides spp. (unidentified) & & + & & & & & & & & & + & & \\
\hline
\end{tabular}

+ Denotes isolation. 


\section{Colony and cell morphology}

Colony size and morphology were of little value in the identification of vaginal isolates except for the characteristic pigmentation of $B$. melaninogenicus and B. asaccharolyticus strains. These species grew better on BM agar than on blood agar; colonies were larger and pigmentation developed earlier. B. melaninogenicus ss. melaninogenicus strains often produced brown rather than black colonies with a paler periphery. There were no consistent differences between the small, pale colonies of the non-pigmented species in the $B$. melaninogenicus/oralis group but the non-pigmented non-saccharolytic strains produced very small $(<0.5 \mathrm{~mm}$ diam.) colonies even after prolonged incubation.

Bacterial-cell morphology was of little discriminatory value. Many strains from the B. melaninogenicus/oralis group appeared similar to B. fragilis-group strains but a little more than half of them were predominantly coccobacillary and a few strains were highly pleomorphic with many distorted and bizarre forms. The asaccharolytic strains were predominantly coccobacillary.

\section{DisCUSSION}

Gram-negative anaerobic bacilli of the Bacteroides group were isolated from swabs taken from the cervix and vaginal fornices of $65 \%$ of the women examined; a heavy growth was obtained from 11 specimens but only a few colonies were isolated from two specimens. This carriage rate is similar to the $65 \%$ found by Sanders et al. (1975) and the $57 \%$ found by Gorbach et al. (1973); it is much greater than the $4 \%$ quoted by Leigh (1976) in a similar population of women attending a family planning clinic, the $4 \%$ in normal women and $1 \%$ in pregnant women found by Lindner et al. (1978), and the $5.4 \%$ and $4 \%$ found by Hurley et al. (1974) and Werner et al. (1978) in pregnant women. These differences in carriage rates may reflect differences between the study populations, such as between pregnant and non-pregnant subjects, or differences in the anaerobic methods of the investigators. In the quantitative study (Lindner et al., 1978), the viable count of Bacteroides spp. in many women may have been below the lower limit of sensitivity of their methods. Vaginal carriage of Bacteroides spp. may vary with the menstrual cycle and could be related to the presence of altered blood (Hurley et al., 1974). The stage of the cycle in the subjects in the present study was not recorded and this aspect of vaginal colonisation could not be assessed. Moreover, the vagina is not a single environment and the differences may in part reflect the sites sampled and the methods of sampling. In the present study, the flora of the cervix and vaginal fornices were considered to represent the high or "true" vaginal flora.

The predominant Bacteroides spp. in the normal vaginal flora in the present study were members of the $B$. melaninogenicus/oralis group; $78 \%$ of isolates belonged to this group. The commonest subgroup of species was the $B$. bivius/disiens complex; $42 \%$ of the B. melaninogenicus/oralis-group isolates 
belonged to this complex and all that were identified fully proved to be $B$. bivius. B. melaninogenicus ss. melaninogenicus $(16 \%)$ and ss. intermedius $(22 \%)$ were the other common species in the group. Only $5 \%$ of the isolates belonged to the $B$. fragilis group and all except one of these isolates were from a single subject. This indicates that the $B$. fragilis group are not part of the normal commensal flora of the vagina. Fusobacterium spp. were not detected, but studies on the gingival flora (Duerden, 1980b) showed that they could be isolated on the selective medium under the same conditions of culture. Asaccharolytic strains, including B. asaccharolyticus were isolated in smaller numbers than the B. melaninogenicus/oralis-group strains; they comprised $12 \%$ of the isolates but were isolated from $54 \%$ of subjects.

The Bacteroides spp. reported in most other studies have not been identified. Lindner et al. (1978) identified their isolates as B. fragilis, B. thetaiotaomicron, B. ovatus and B. vulgatus but did not detect strains of the B. melaninogenicus/oralis group. This may reflect differences in the anaerobic procedures or the selective medium used; the medium contained neomycin sulphate 1000 $\mathrm{mg} /$ litre and results in antibiotic-disk resistance tests have shown that the $B$. melaninogenicus/oralis group is less resistant to neomycin than the $B$. fragilis group (Duerden et al., 1976; Holbrook, Duerden and Deacon, 1977; Duerden et al., 1980). B. fragilis, B. thetaiotaomicron, B. melaninogenicus and B. oralis were the four strains isolated by Werner et al. (1978) from normal pregnant women but $B$ oralis was the predominant anaerobic species isolated from the uterine cavity, cervical canal or vagina of puerperal women; $42 \%$ of these patients harboured clinically important anaerobes.

The predominance of the B. melaninogenicus/oralis group amongst the Bacteroides spp. in the normal vaginal flora and the absence of significant numbers of $B$. fragilis-group isolates contrasts with the preponderance of the $B$. fragilis group in the faeces where the B. melaninogenicus/oralis group is not commonly part of the normal flora. (Duerden, 1980a).

The common vaginal species were, however, similar to the oral species isolated from subgingival plaque; in a parallel study (Duerden, 1980b) the commonest oral species were $B$. oralis, $B$. melainogenicus ss. melaninogenicus and ss. intermedius. The similarity in the identity of isolates from the two sites may reflect similarities in the physiological environments. A comparison of saliva with cervical secretions shows that the $p \mathrm{H}$, osmolarity, carbohydrate and enzyme content, in particular the amylase content, are similar (Jordan and Singer, 1976; Mason and Chisolm, 1975).

Pigmented strains of $B$. melaninogenicus and $B$. asaccharolyticus were isolated from nine $(45 \%)$ of the 20 subjects in the present series. This is a lower carriage rate than Burdon (1928) found; he isolated "B. melaninogenicus" from $28(80 \%)$ of the 35 vaginal specimens from normal women.

The present study has shown that gram-negative anaerobic bacilli are common but not universal commensals of the cervix and vaginal fornices. The common species found in the normal vaginal flora were members of the $B$. melaninogenicus/oralis group and these species were readily isolated and identified by the conventional methods used in this study. 


\section{SUMmary}

The occurrence of gram-negative anaerobic bacilli in the normal vaginal flora was studied in 20 normal healthy women attending a family-planning clinic. A swab was taken from the cervix and posterior fornix and Bacteroides spp. were isolated on a selective medium from $13(65 \%)$ subjects. A heavy growth of Bacteroides was obtained from 11 specimens but only a few colonies were isolated from two specimens. Where possible, 10 representative colonies from each subject were studied and 113 isolates were identified by conventional bacteriological tests. Most isolates $(78 \%$ ) belonged to the $B$. melaninogenicus/oralis group. The commonest species identified in this group were the $B$. bivius/disiens complex $(42 \%)$, B. melaninogenicus ss. intermedius $(22 \%)$ and ss. melaninogenicus $(16 \%)$. Asaccharolytic strains were isolated in smaller numbers from $54 \%$ of subjects, but only five strains of the B. fragilis group were isolated from two subjects; fusobacteria were not detected.

I am grateful to Professor J. G. Collee for encouragement and advice throughout these investigations, to Mrs Julia Faulkner for her invaluable technical assistance and to Dr M. Griffiths, Sheffield Family Planning Clinic, who collected the specimens.

\section{REFERENCES}

BURDON, K. L. 1928. Bacterium melaninogenicum from normal and pathologic tissue. J. inf. Dis., 42, 161.

Collee, J. G., Watt, B., Fowler, E. B. And Brown, R. 1972. An evaluation of the Gaspak system in the culture of anaerobic bacteria. J. appl. Bact., 35, 71 .

Deacon, A. G., Duerden, B. I. AND Holbrook, W. P. 1978. Gas-liquid chromatographic analysis of metabolic products in the identification of Bacteroidaceae of clinical interest. $J$. med. Microbiol., 11, 81 .

DuERDEN, B. I. 1980a. The isolation and identification of Bacteroides spp. from the normal human faecal flora. J. med. Microbiol., 13, 69.

DUERDEN, B. I. $1980 b$. The isolation and identification of Bacteroides spp. from the normal human gingival flora. J. med. Microbiol., 13, 89.

Duerden, B. I., Collee, J. G., Brown, R., Deacon, A. G. And Holbrook, W. P. 1980. A scheme for the identification of clinical isolates of gram-negative anaerobic bacilli by conventional bacteriological tests. J. med. Microbiol., 13, in press.

Duerden, B. I., Holbrook, W. P., Collee, J. G. ANd Watt, B. 1976. The characterization of clinically important gram-negative anaerobic bacilli by conventional bacteriological tests. J. appl. Bact., 40, 163.

Finegold, S. M. 1977. Anaerobic bacteria in human disease, Academic Press: London, p. 32.

Gorbach, S. L., Menda, K. B., Thadepalli, H. and Keith, L. 1973. Anaerobic microflora of the cervix in healthy women. Am. J. Obstet. Gynec., 117, 1053.

Holbrook, W. P., Duerden, B. I. AND Deacon, A. G. 1977. The classification of Bacteroides melaninogenicus and related species. J. appl. Bact., 42, 259.

Hurley, R., Stanley, V. C., Leask, B. G. S. ANd de Louvois, J. 1974. Microflora of the vagina during pregnancy, In The normal microbial flora of man, edited by F.A. Skinner and J. G. Carr, Society for Applied Bacteriology, symposium series 3. Academic Press: London, p. 155.

Jordan, J. A. And Singer, A. 1976. The cervix. Saunders: London.

LEIGH, D. A. 1976. Bacteroides infections: diagnosis and treatment. In Selected topics in clinical bacteriology, edited by J. de Louvois. Balliere Tindal: London, p. 129. 
Lindner, J. G. E. M., Plantema, F. H. F. and Hoogkamp-Korstanje, J. A. A. 1978. Quantitative studies of the vaginal flora of healthy women and of obstetric and gynaecological patients. J. med. Microbiol., 11, 233.

Mason, D. U. and Chisolm, D. M. 1975. Salivary glands in health and disease. Saunders: London.

Mead, P. B. and Louria, D. B. 1969. Antibiotics in pelvic infections. Clin. Obstet. Gynec., $12,219$.

MöLLER, Å. J. R. 1966. Microbiological examination of root canals and periapical tissues of human teeth: methodological studies. Odont. Tid., 74 (suppl.), p.365.

Neary, M. P., Allen, J., Okubadejo, O. A. and Payne, D. J. H. 1973. Preoperative vaginal bacteria and postoperative infections in gynaecological patients. Lancet, 2, 1291.

Sanders, C. V., Mickal, A., Lewis, A. C. AND Torres, J. 1975. Anaerobic flora of the endocervix in women with normal versus abnormal Papanicolaou (Pap) smears. Clin. Res., 23, 30A.

Werner, H., Lang, N., Kraseman, C., Tolkmitt, G. and Feddern, R. 1978. Epidemiology of anaerobic infections of the female genito-urinary tract, and preliminary results of therapy with erythromycin. Curr. med. res. Opin., 5, suppl. 2, 52. 